\title{
Analysis of Arbitrary Reflector Antennas Applying the Geometrical Theory of Diffraction Together with the Master Points Technique
}

\author{
María Jesús Algar, Jose-Ramón Almagro, Javier Moreno, \\ Lorena Lozano, and Felipe Cátedra \\ Departamento de Ciencias de la Computación, Universidad de Alcalá, Alcalá de Henares, 28871 Madrid, Spain \\ Correspondence should be addressed to María Jesús Algar; chus.algar@uah.es
}

Received 2 November 2012; Accepted 4 January 2013

Academic Editor: Miguel Ferrando

Copyright ( $) 2013$ María Jesús Algar et al. This is an open access article distributed under the Creative Commons Attribution License, which permits unrestricted use, distribution, and reproduction in any medium, provided the original work is properly cited.

An efficient approach for the analysis of surface conformed reflector antennas fed arbitrarily is presented. The near field in a large number of sampling points in the aperture of the reflector is obtained applying the Geometrical Theory of Diffraction (GTD). A new technique named Master Points has been developed to reduce the complexity of the ray-tracing computations. The combination of both GTD and Master Points reduces the time requirements of this kind of analysis. To validate the new approach, several reflectors and the effects on the radiation pattern caused by shifting the feed and introducing different obstacles have been considered concerning both simple and complex geometries. The results of these analyses have been compared with the Method of Moments (MoM) results.

\section{Introduction}

Reflector antennas have been used since 1888, when electromagnetic waves were discovered by Hertz. Although these antennas are very common in radar systems, they were not in widespread use until the Second World War [1,2]. Thereafter, the demand of reflector antennas has grown in several fields such us radio astronomy, microwave communication, space communications, and radio links working at millimeter frequencies [3].

This increasing interest is due to the pencil-beam-shaped pattern and the possibility to configure the radiation pattern of a reflector by changing the shape and/or its feed array. In this way, the characteristics of its radiation pattern, for example, the effective area or the relationship between the main and the secondary lobes, can be adjusted to achieve specific requirements. For that reason, these antennas have a wide range of applications, like satellite communications and radar systems, which demand an accurate and efficient analysis of complex reflectors.
The shape and directivity of the radiation pattern of a reflector antenna depend on the electric and magnetic field distribution on its aperture. Therefore, the configuration of its radiation pattern is closely linked with its geometrical model. Consequently, according to the geometry of the reflector, these antennas can be classified into dihedral, cylindrical, parabolic, elliptical, spherical, offset, and hyperbolic reflectors. The most commonly used is the parabolic reflector, due to its highly directive radiation pattern.

High frequency techniques, such us physical optics (PO) [4] or the Geometrical Theory of Diffraction (GTD) [5], have been used extensively in the literature [6-11] to evaluate the radiation pattern of reflector antennas. For instance, in [7], it is remarked that the GTD analysis provides good accuracy in the results for the prediction of the main beam and sidelobes of a parabolic reflector. Furthermore, it has been demonstrated that the physical optics radiation integral provides an accurate solution for predicting the far field radiated by reflector antennas. However, due to the large electrical dimensions of these structures, the PO integral accounts for most of the 
computation time. For that reason, [11] presents a domain decomposition algorithm to compute the radiation pattern of a finite aperture with a high computational efficiency. In this regard, a new technique to compute the $\mathrm{PO}$ integral in a more efficient way is presented in [6]. The procedure presented in [8] is based on a series of Fourier transforms of an aperture distribution which takes into account the curvature of the surface. Additionally, the aperture blockage effect on the pattern of a quadruped supported primary-feed parabolic reflector is evaluated in [9], where the scattering process is obtained as an interaction between the reflector structure and the aperture-blocking feed together with its supporting struts.

This paper is focused on the problem of analyzing the radiation pattern of arbitrarily shaped reflector antennas applying GTD. The electrical field in a large number of points sampled on the aperture of the reflector is obtained. That is, the ray tracing algorithm is calculated for each observation point, and this process spends too much CPU time. Therefore, GTD has been combined with a new technique, named Master Points, to accelerate the calculation of the electromagnetic field in this kind of analysis. The speed-up is possible since this method reduces the number of times in which it is necessary to calculate the ray tracing to obtain the electromagnetic field in a given number of observation points or directions.

In the ray tracing algorithm, the Conjugate Gradient Method (CGM) [12] is applied to obtain the reflection points on the reflector surfaces. This method minimizes a cost function which depends on the distance between the source and any given observation point or direction. Usually, the geometric model of a complex body is composed of flat and convex surfaces. However, the geometrical model of a parabolic reflector is composed of concave instead of convex surfaces. Therefore, a new distance function to analyze a concave surface is proposed.

This paper is organized as follows. Section 2 reviews the geometric and electromagnetic analysis of a canonical parabolic reflector. The ray tracing algorithm used to compute the radiation pattern of these antennas and the improvements done in this process are presented in Sections 3 and 4 , respectively. Section 5 is focused on the application of the Master Points technique to perform this analysis. Finally, Section 6 shows several cases of study to validate this new approach, including the analysis of the blockage in a parabolic reflector caused by an obstacle.

\section{Application of the Ray-Tracing Algorithm to Analyze an Arbitrarily Shaped Reflector}

As it has been outlined in the Introduction section, when the radiation pattern of a reflector antenna is computed, the first step is to calculate the electromagnetic field distribution on a very dense mesh of points sampled on its aperture. The main purpose of this step is to perform it as fast as possible. To compute the ray tracing on a complex structure, two situations are distinguished depending on the geometry of the object:

(i) geometry composed of flat surfaces: in this case, the search for reflection points on the geometry is

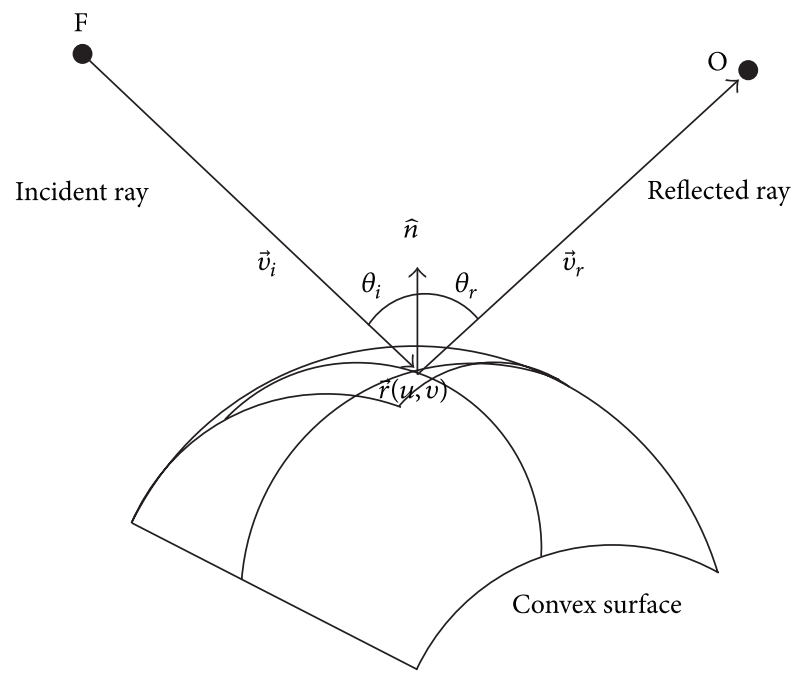

FIGURE 1: Simple reflection on a convex surface.

performed analytically using the Image Theory (IT) [13];

(ii) geometry composed of curved surfaces: regarding arbitrary surfaces like NURBS $[14,15]$, the search for reflection points becomes more complicated, and minimization methods must be applied; in particular, the CGM [12], which minimizes a cost function, is used in this paper; additionally, the minimization process for a convex surface and for a concave surface is different, the following sections describe the differences for both surfaces.

2.1. Reflection Point on Convex Surfaces. The search for reflection point on a geometry composed of convex surface is complicated, as the Image Theory cannot be applied. A simple schema describing this situation, for a given source and observation point, is shown in Figure 1. To determine the point within the area on which the reflection will take place, the CGM minimizes a cost function. This function is different depending on whether the near field or the far field is analyzed. For instance, in the analysis of the near field, this cost function is expressed as follows:

$$
d(u, v)=d_{i}(u, v)+d_{r}(u, v)=\left|\vec{v}_{i}(u, v)\right|+\left|\vec{v}_{r}(u, v)\right|,
$$

where $d_{i}(u, v)$ is the distance between the source and the candidate reflection point and $d_{r}(u, v)$ is the distance between the candidate reflection point and the observation point. Hence, $\vec{v}_{i}$ is the vector from the source to the candidate reflection point, and $\vec{v}_{r}$ is the vector from the candidate reflection point to the observation point.

The minimization process of the CGM method implements the following steps.

(1) First of all, a seed point over the convex surface is selected.

(2) Then, the cost function and its partial derivatives are evaluated in this point. 
(3) If the partial derivatives are null, the minimum of the function has been reached. Consequently, the search process finishes with that seed point as the final solution: the reflection point.

(4) Otherwise, the seed point is shifted over the curve according to the direction given by the partial derivatives.

(5) This last seed point is evaluated repeating step (2).

This iterative process will be completed when the minimum of the function is found or when it exceeds a certain number of iterations.

This shift from one seed point to another is done according to the partial derivatives of (1), which are given by the following expressions:

$$
\begin{aligned}
\frac{\partial d(u, v)}{\partial u} & =\frac{\partial d_{i}(u, v)}{\partial u}+\frac{\partial d_{r}(u, v)}{\partial u} \\
& =\left[\vec{v}_{i}(u, v)+\vec{v}_{r}(u, v)\right] \cdot \vec{r}_{u}(u, v), \\
\frac{\partial d(u, v)}{\partial v} & =\frac{\partial d_{i}(u, v)}{\partial v}+\frac{\partial d_{r}(u, v)}{\partial v} \\
& =\left[\vec{v}_{i}(u, v)+\vec{v}_{r}(u, v)\right] \cdot \vec{r}_{v}(u, v),
\end{aligned}
$$

where $\vec{r}_{u}(u, v)$ and $\vec{r}_{v}(u, v)$ are the partial derivatives, with respect to the parametric coordinates $u$ and $v$ of the reflection point.

When the far field is analysed, the CGM minimizes the following cost function:

$$
\begin{aligned}
d(u, v) & =d_{i}(u, v)+d_{r}(u, v) \\
& =\left|\vec{v}_{i}(u, v)\right|+(D-\widehat{v} \cdot \vec{r}(u, v)),
\end{aligned}
$$

where $d_{i}(u, v)$ is the distance between the source and the candidate reflection point, $d_{r}(u, v)$ is the distance between the candidate reflection point and a perpendicular plane to the observation direction, $\vec{v}_{i}(u, v)$ is the vector from the source to the candidate reflection point, $\widehat{v}$ is a unitary vector which determines the observation direction, $D$ is the independent term of the equation that defines the perpendicular plane to the observation direction, and $\vec{r}(u, v)$ is the candidate reflection point on the surface.

The minimization process and the associated shift of the seed point are performed as in the previous case. The partial derivatives of (3) are given by the following expressions:

$$
\begin{aligned}
\frac{\partial d(u, v)}{\partial u} & =\frac{\partial d_{i}(u, v)}{\partial u}+\frac{\partial d_{r}(u, v)}{\partial u} \\
& =\left[\vec{v}_{i}(u, v)-\widehat{v}\right] \cdot \vec{r}_{u}(u, v), \\
\frac{\partial d(u, v)}{\partial u} & =\frac{\partial d_{i}(u, v)}{\partial u}+\frac{\partial d_{r}(u, v)}{\partial u} \\
& =\left[\vec{v}_{i}(u, v)-\widehat{v}\right] \cdot \vec{r}_{v}(u, v) .
\end{aligned}
$$

Once the reflection point has been found on the surface, a shadowing test is conducted to affirm that neither reflected

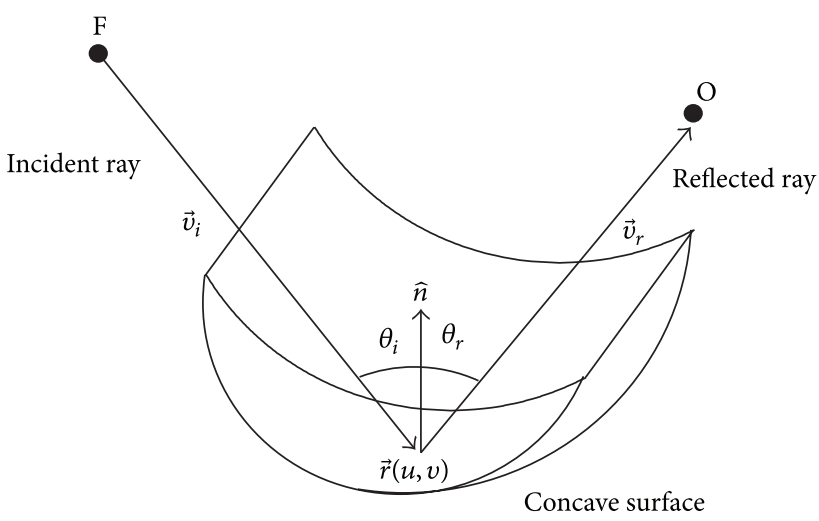

FIgURE 2: Simple reflection on a concave surface.

nor incident paths are hidden by any surface. Likewise, the reflection point must be located on the curve, and Snell's law must be satisfied at that point [16] as follows:

$$
\widehat{k}_{i} \operatorname{sen} \theta_{i}=\widehat{k}_{r} \operatorname{sen} \theta_{r} .
$$

If any of these conditions are not satisfied, the reflection point found by the CGM is not the right solution and the algorithm will need to find another point.

2.2. Reflection Point on Concave Surfaces. The cost functions considered in this case, for either the near or the far field analysis, are the same as in the case of a convex curve, and they are described by expressions (1) and (3), respectively. Figure 2 shows this case of study schematically.

Instead of the search process for the reflection point on a convex surface, in which the CGM looks for a minimum of the cost function, in this case, the CGM looks for both a minimum and a maximum of the function presented previously.

Before this process starts, a sampling of the curve on which the reflection point has to be found, along the parametric coordinates $u$ and $v$, is performed. Each of these samples is considered as a seed point for the search of a minimum or a maximum. As in the minimization process over a convex surface, the shifted over the curve is done according to the sign of the derivative functions, expressions (2) or (4), depending on the case (the near field or the far field, resp.). Once the solution point is found, if it belongs to the curve, satisfies Snell's law and none of the incident and reflected paths are shadowed by any obstacle, it can be concluded that the reflection point has been found on the concave surface.

In order to better understand the minimization process implemented by CGM, Figure 3 shows a schema applicable to convex or concave curves.

\section{Improvements Done over the Ray-Tracing Algorithm}

The geometrical model of reflector systems is mainly composed of curved surfaces, in particular, concave surfaces. However, in the analysis of the radiation pattern of antennas on board complex structures, the geometrical models used 


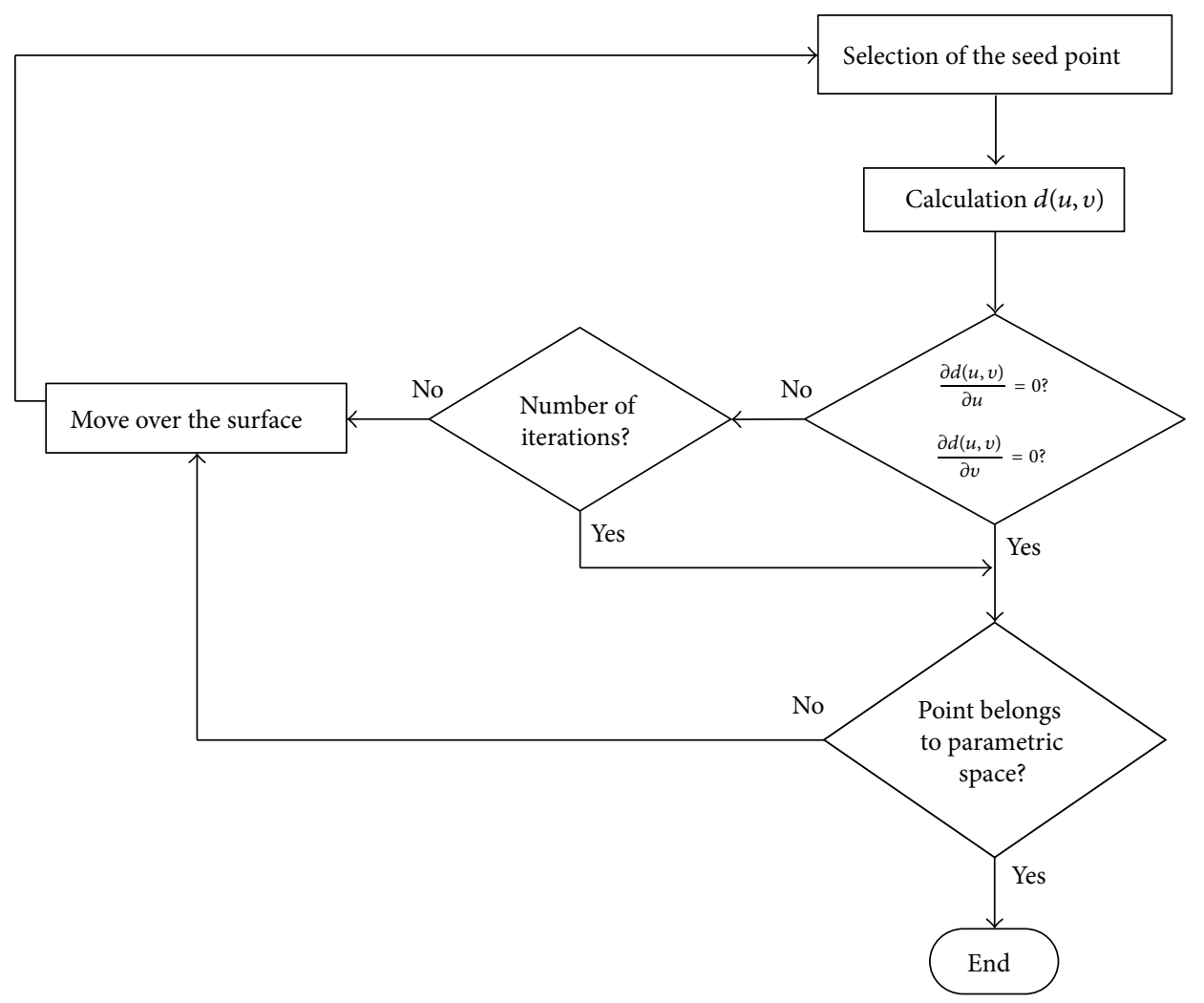

FIGURE 3: Flow chart for the minimization process.

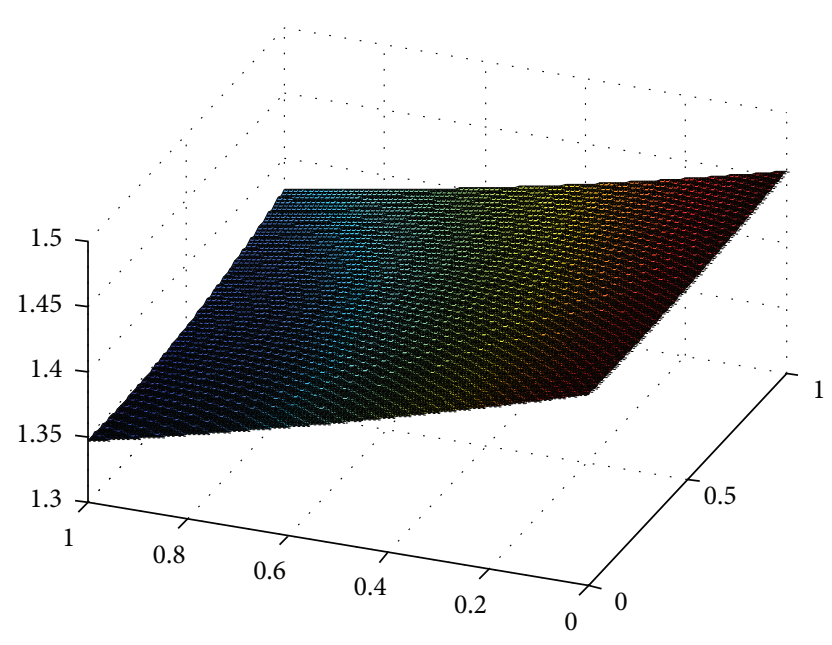

FIGURE 4: Representation of function (1) over a concave surface.

correspond to bodies such as ships, tanks or aircrafts. Most of these objects are composed of flat surfaces or convex surfaces.

As it is mentioned earlier, the process followed to search reflection points is different for concave and convex surfaces. The CGM minimizes a cost function which depends on the sum of the distances between the source and the candidate reflection point and between this point and the observation point or direction, expressions (1) or (3), respectively [13].
Nevertheless, the analysis of concave surfaces seeks either a maximum or a minimum of the same function.

To ensure that the minimization or maximization of the distance function is adequate, this function must have an absolute minimum or maximum. That is, the function must not present a smooth variation between their values, as in this case, the algorithm would fail to converge towards an inexistent solution point.

If the distance functions of expressions (1) and (3) are represented on a concave surface, the obtained plot is very similar to the one shown in Figure 4.

As it can be observed in Figure 6, the function has very smooth variation, and it does not present any absolute minimum or maximum. Therefore, both functions (1) and (3) on the concave curves are not adequate to ensure that the CGM algorithm will converge towards the right reflection point. Even if the seed point is very close to the real reflection point, the CGM is not able to find that point minimizing or maximizing the function in Figure 4.

In order to clarify that the minimization process with this function is not possible, the parabolic reflector with $1 \mathrm{~m}$ diameter, shown in Figures 5 and 6 , is analyzed. The horn feed is located on the focus $(0,0,0.4)$, and a set of observation points are arranged in a straight line. The reflection points obtained are those that appear on the surface of the reflector.

As was demonstrated in Section 2, the parabolic reflector transforms a spherical wave into a plane wave. This means that the rays from the reflection points must be parallel to the radiation axis of the reflector. This affirmation implies that 


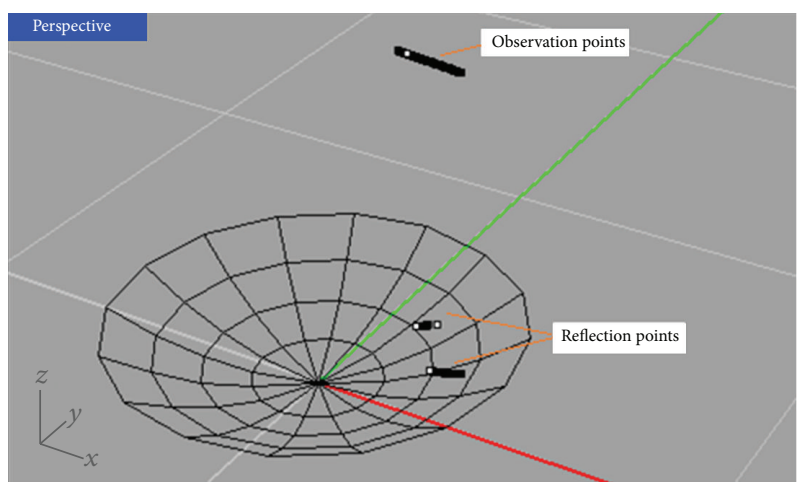

FIGURE 5: Perspective view of the simple reflection points obtained with function (1) or (3).

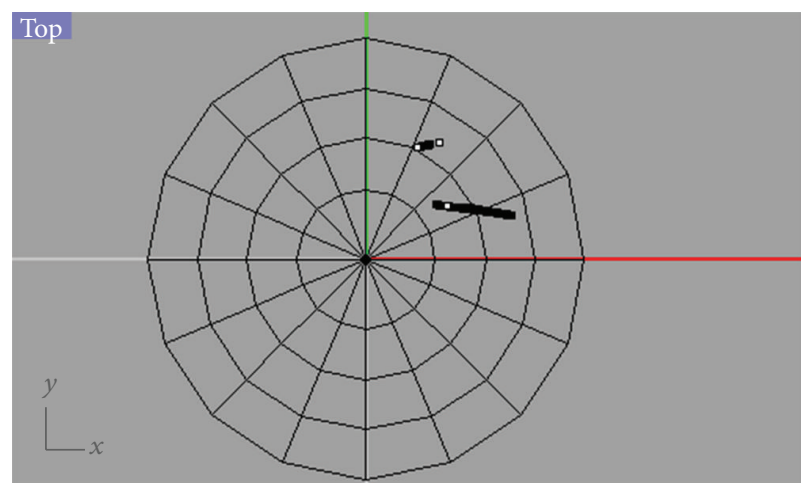

Figure 6: Top view of the simple reflection points obtained with function (1) or (3).

the reflection points will be the projection of the observation points on the reflector surface. However, the reflection points shown in Figures 5 and 6 are not the projection of the observation points on the surface of the reflector.

For that reason, the CGM is not able to find the minimum or maximum absolute of the cost function in the appropriate surface. Instead, it finds a minimum or a maximum in a nearby surface on which the reflection does not take place. As a result, it is necessary to establish another cost function which presents an absolute maximum or minimum to ensure that the algorithm can find the suitable solution.

In the first step, it could be thought that, as any reflection on any type of surface, concave or convex, must satisfy Snell's law, this condition can be set as the function to be explored by CGM. Hence, this function will take the form of the following expression:

$$
\left|\widehat{k}_{i} \otimes \widehat{k}_{r}\right|
$$

where $\widehat{k}_{i}$ is the unitary incident vector, and $\widehat{k}_{r}$ is the unitary reflection vector.

Figure 7 shows the plot of expression (6) on a concave surface.

Comparing Figures 4 and 7, it can be concluded that the new function shown in Figure 7 presents a higher degree of variation between their values than Figure 4 .

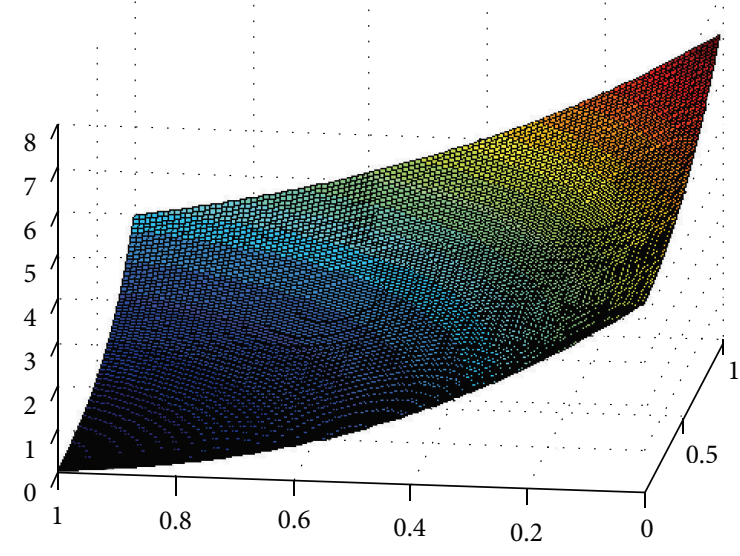

FIGURE 7: Graphical representation of the Snell law.

However, despite this growth in the variation, the adequate convergence of the algorithm is not guaranteed because the function does not present either an absolute maximum or minimum, yet. It presents multiple local maximum or minimum points. This is not enough to establish Snell's law as the new cost function to look for reflection points in this type of surfaces.

In order to guarantee that a point on a surface is a reflection point, one more condition must be satisfied, the incident vector, the observation vector, and the normal in that point must be coplanar; that is, all of them should belong to the same plane. If this condition is added to expression (6), the cost function is transformed into this new expression:

$$
\left|\widehat{n} \cdot \widehat{k}_{i}-\widehat{n} \cdot \widehat{k}_{r}\right|+\left|\widehat{k}_{i} \otimes \widehat{k}_{r}\right|
$$

where $\widehat{n}$ is the normal to the surface in the reflection point.

If the values taken from expression (7) are represented on a concave surface, the graph shown in Figure 8 is obtained.

Comparing Figure 8 with Figures 4 and 7, it is clear that the last one exhibits the best features for its minimization or maximization with an absolute minimum or maximum. Therefore, it can be deduced that this function will make it possible to carry out the search of reflection points on concave surfaces in a satisfactory way.

Considering again the example of the parabolic reflector of $1 \mathrm{~m}$ diameter shown in Figures 5 and 6, now, the same test is done with function (7). If the CGM works with this function, it can be asserted that it can find the points of the simple reflection on the concave curves, as shown in Figure 9.

Figure 10 shows how, in this case, the reflection points are the projection of the observation points on the curves of the reflector. The rays reflected on the reflector are parallel to the $z$-axis, and then the spherical wave impacting on its surface is transformed into a plane wave, confirming the good behavior of the reflector.

In this way, the correct performance of the CGM has been demonstrated, working with the new cost function, for reflection points search on concave surfaces. 


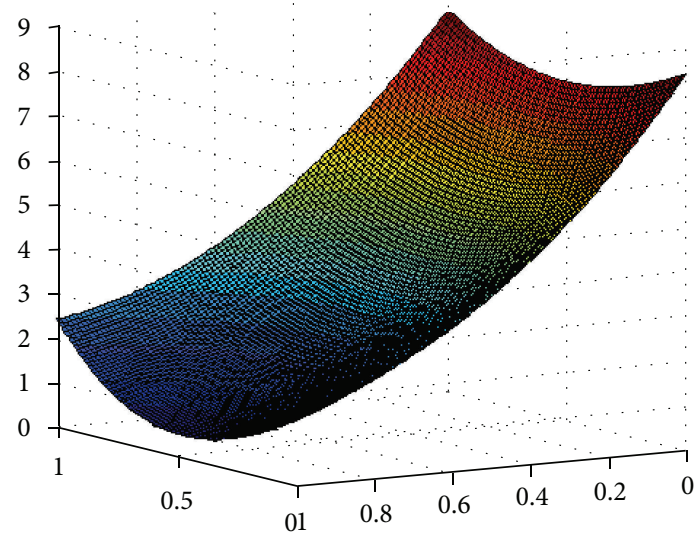

FIGURE 8: Graphical representation of function (7) over a concave surface.

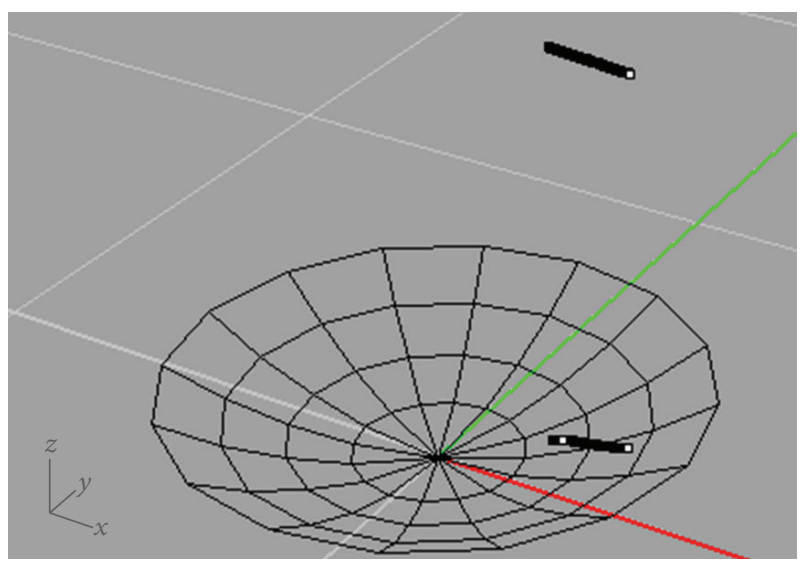

Figure 9: Perspective view of the simple reflection points obtained with function (7).

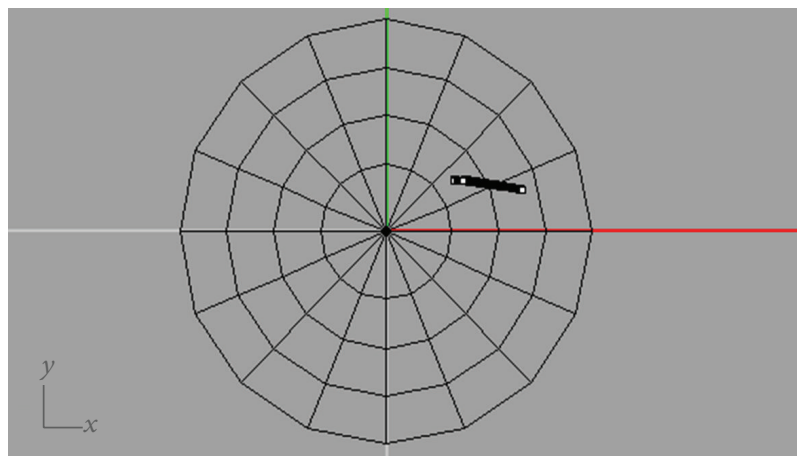

FIGURE 10: Top view of the simple reflection points obtained with function (7).

\section{Master Points Algorithm to Analyze Reflector Antennas}

As it has been mentioned previously, the analysis of the radiation pattern of reflector structures can be done calculating the electromagnetic fields at the aperture and then transforming the near field to the far field [7]. To perform this analysis,

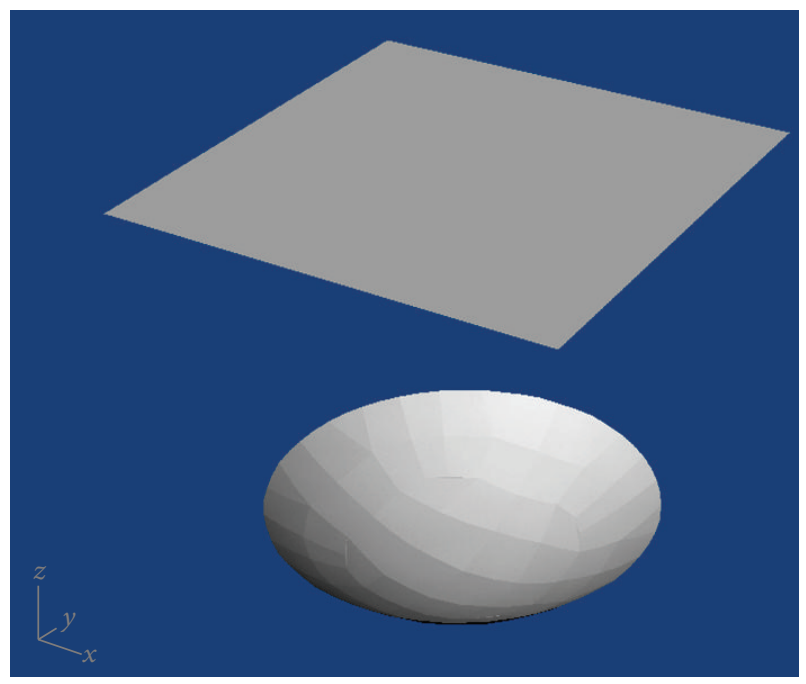

FIGURE 11: Geometrical model of a single reflector with its observation surface.

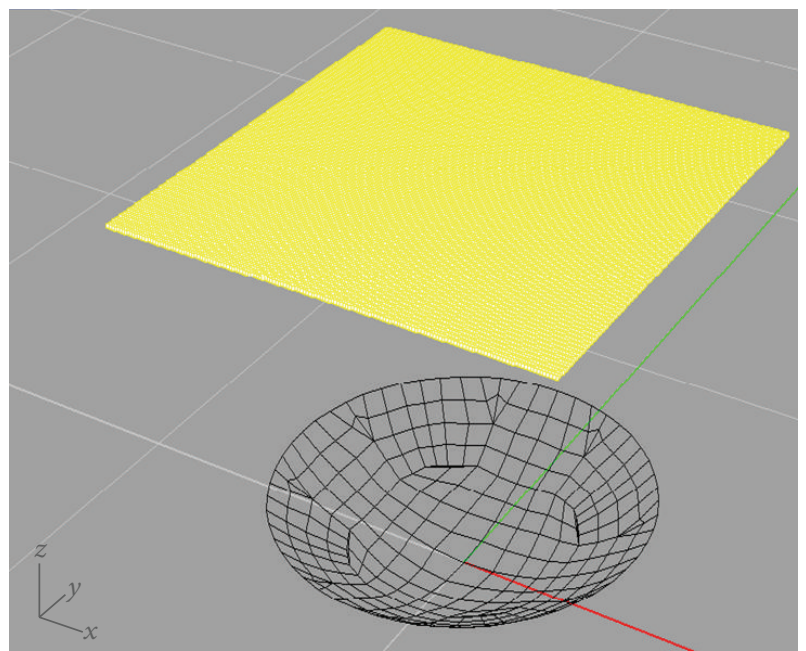

FiguRE 12: Observation surface sampled at $\lambda / 3$.

a fictitious surface, like the one shown in Figure 11, must be placed on the aperture of the reflector, to cover it completely. This surface must be perpendicular to the radiation axis of the reflector. It is sampled, obtaining a huge amount of observation points in which the near field will be obtained (Figure 12).

Once the near field has been calculated on the set of observation points, applying the high frequency technique GTD, most of the CPU time is spent obtaining the ray tracing for each observation point. Thus, the new algorithm Master Points has been applied to speed up this process. Finally, the transformation of the near field to far field is applied, obtaining the radiation pattern of the antenna.

To obtain the near field on a sampled plane of points, the Master Points technique makes a compartmentalization of all points, depending on the existence of the ray tracing. Figure 13 shows an example of a plane of observation points. This 


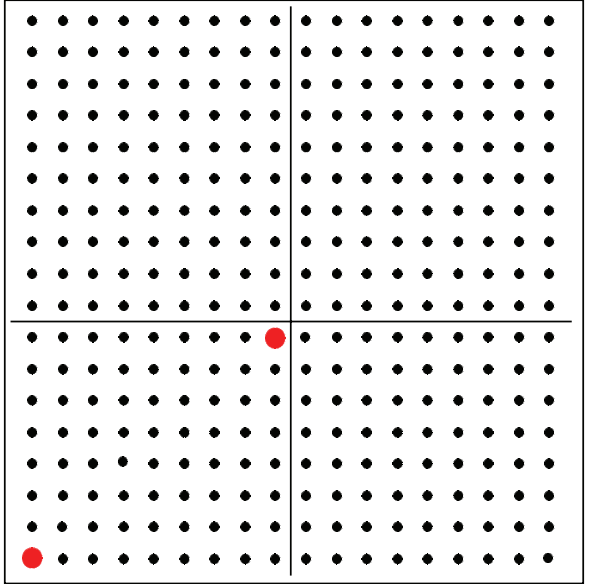

FIGURE 13: Observation plane divided into 4 quadrants.

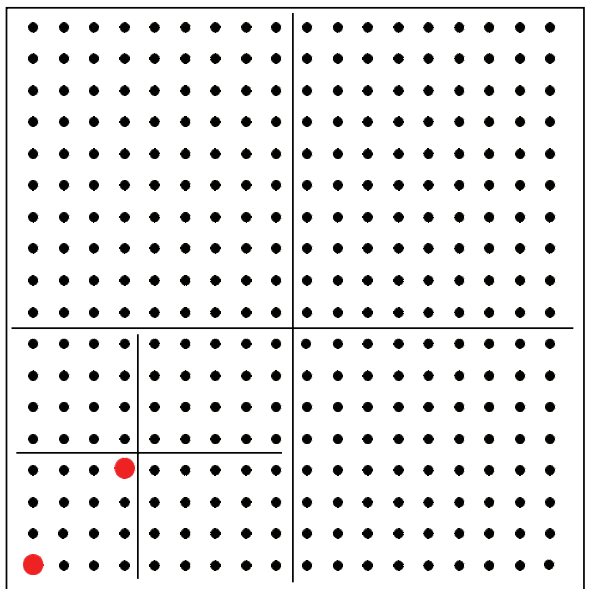

FIGURE 14: Division process.

plane is divided into 4 quadrants, and the algorithm begins to analyze the quadrant located at the left button corner.

This analysis tests if there is ray tracing for both external points (red points). If so, a group, containing all the points in this quadrant, is formed, and the next quadrant, located at the right button corner, is analyzed in the same way. However, if this is not the case, a new division is done as shown in Figure 14. This iterative process continues until the whole plane has been evaluated or the limit number of divisions has been reached. As a result of our experience in the application of this technique to compute the radiation pattern in a vast number of observation points or directions, 4 is a good value for the depth limit that can let us obtain accuracy in the results diminishing the CPU time.

Once a group is formed, several sampled points are selected to obtain the near field only in these points. It is important to know that the accuracy of results depends on the way this selection is done. For example, if the group of $7 \times 7$ points shown in Figure 15 has been formed, the results will be better if 4 samples instead of 3 are selected in each direction, because the ray tracing is obtained in more samples.
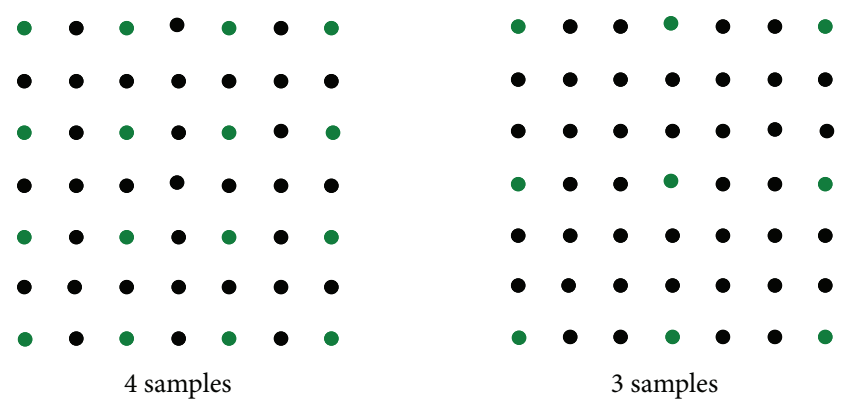

FIGURE 15: Taking samples in a group.

Finally, to obtain the near field in all observation points, an interpolation method is applied. That is, for several points (samples), the ray tracing and the near field have been calculated. Applying an interpolation method to these values of near field, the near field in all the observation points of the group is obtained. Instead of an interpolation method, an approximation method is applied since it reduces the total error, fitting better the sample's values. In particular, the approximation method used is $2 \mathrm{D}$ least square minimization.

\section{Results}

In order to validate the improvement developed in this paper in the analysis of reflection on concave surfaces, an extensive study on the calculation of the radiation pattern of reflector structures in multiple situations is presented in the sequel. The results of this analysis have been compared with MoM results.

In the first section, the analysis of a single reflector has been performed to study the effects introduced in the radiation pattern by the shift of the feed. In the second section, the radiation pattern of a single reflector considering a feed array is shown. To conclude, several obstacles have been placed on the antenna directivity to determine the effect produced in the radiation pattern of the antenna.

5.1. Feed's Shift. This section presents the analysis of the variations that experiment the radiation pattern of a reflector antenna as the position of feeds is modified, by applying the combination of GTD with the Master Point method discussed earlier.

Figure 16 shows the geometric model of the antenna considered in this study. It is a parabolic reflector with $1 \mathrm{~m}$ diameter and its focus at $(0.0,0.0,0.4)$. The observation surface, located over the reflector aperture, has been sampled at $\lambda / 3$. This means that at $10 \mathrm{GHz}, 14,400$ observation points are obtained.

This reflector has been fed with a rectangular horn that presents the radiation pattern shown in Figure 17.

First of all, to analyze the effect introduced by the shift of the feed, the radiation pattern of the single reflector shown in Figure 16 is obtained, locating the horn at its focus point $(0.0,0.0,0.4)$. The results for the polar component applying GTD combined with the Master Points method have been 


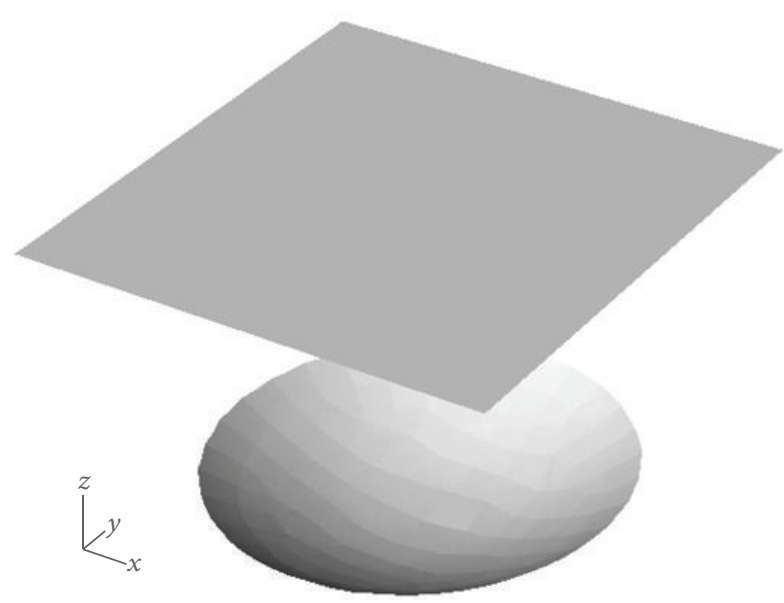

FIGURE 16: Single reflector with observation surface.

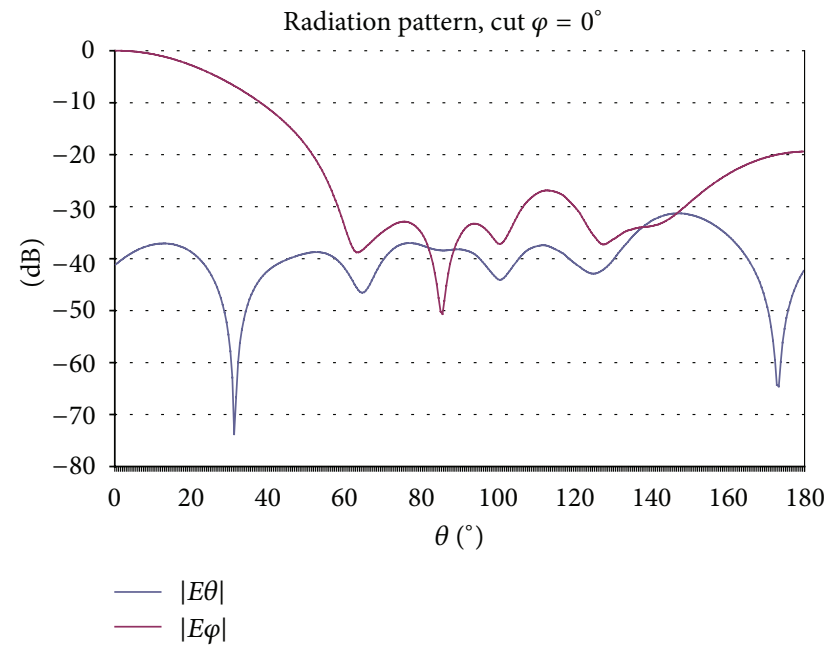

FIGURE 17: Radiation pattern of the rectangular horn.

compared with the rigorous technique MoM, showing a good agreement as depicted in Figure 18.

The main lobe is located at $\varphi=0^{\circ}$ and $\theta=0^{\circ}$ because the horn is located at the focus of the reflector.

5.1.1. $x$-Axis. For this case of study, the horn has been moved from the focus point to $(0.02,0.0,0.4)$, over the $x$-axis. The new schema is represented in Figure 19.

Figure 20 shows the comparison between GTD-Master Points and MoM at a frequency of $10 \mathrm{GHz}$. A cut in $\varphi=0^{\circ}$ and sweep from $\theta=-70^{\circ}$ to $\theta=70^{\circ}$ are represented. The graph shows good accuracy for the GTD-Master Point method.

In the first case, the main lobe was located at $\varphi=0^{\circ}$ and $\theta=0^{\circ}$. However, when the horn is moved over the $x$-axis, the main lobe experienced a slight offset. As shown in Figure 20, the main lobe is approximately located at $\varphi=0^{\circ}$ and $\theta=-2^{\circ}$.

5.1.2. $x y$-Axis. It is also interesting to know what happens with the radiation pattern of the reflector when the horn is moved over the $x$-axis and the $y$-axis. The geometrical model

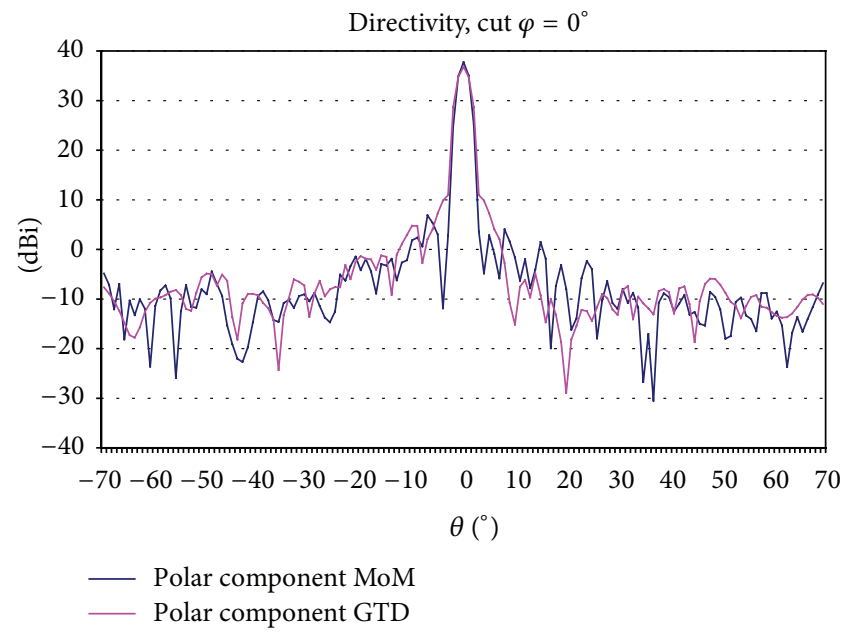

FIgure 18: Polar component obtained with MoM and GTD, cut in $\varphi=0^{\circ}$.

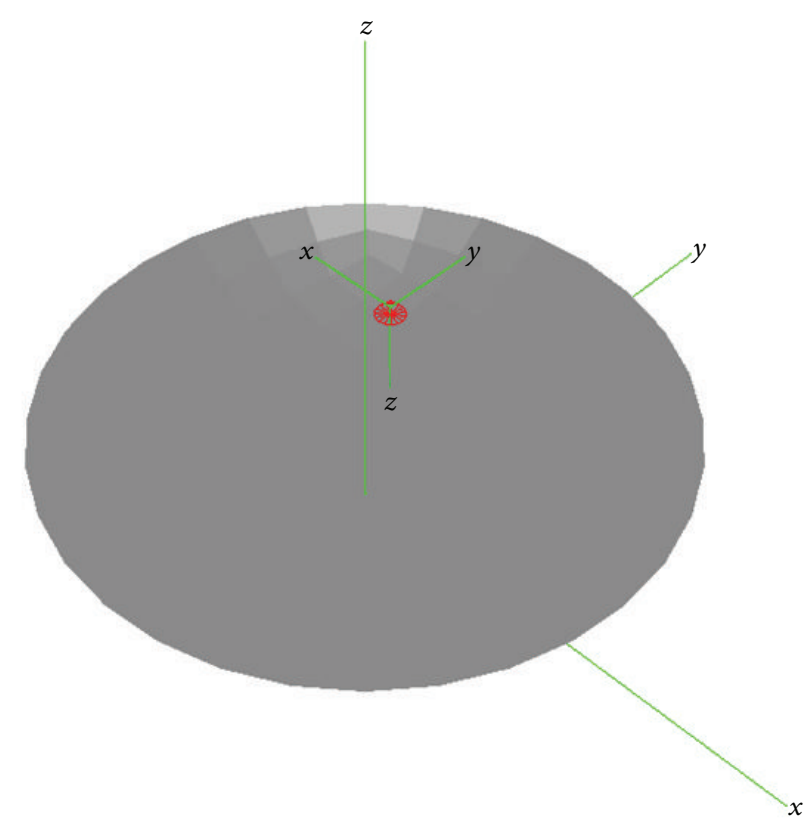

Figure 19: Single reflector with the horn shifted.

considered in this case is shown in Figure 21, where the horn has been placed at the point $(0.02,0.02,0.4)$.

Results for the polar component are presented, in Figure 22. In this case, the main lobe has been shifted to $\varphi=45^{\circ}$ and $\theta=-3^{\circ}$, approximately. The frequency of the simulation is $10 \mathrm{GHz}$.

5.2. Feed a Single Reflector with an Array. Once the effect caused by the shift of the feed of a reflector from its focal position has been studied, it is interesting to see what happens with the directivity of the antenna when the reflector is fed by an array of horns. This is analyzed for the following two cases.

(i) In the first one, a linear array consisting of three horns located over the $x$-axis is considered. 


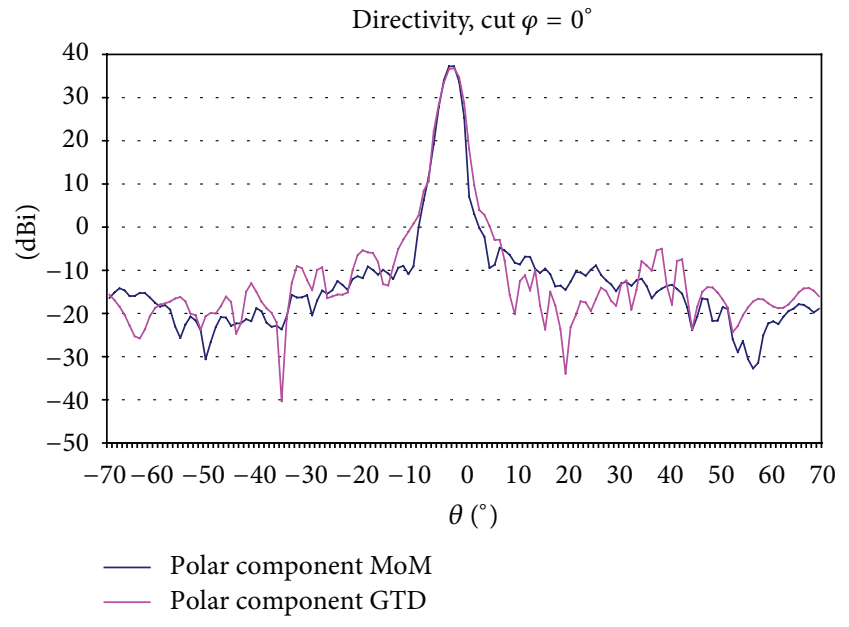

Figure 20: Polar component obtained with MoM and GTD, cut in phi $=0$.

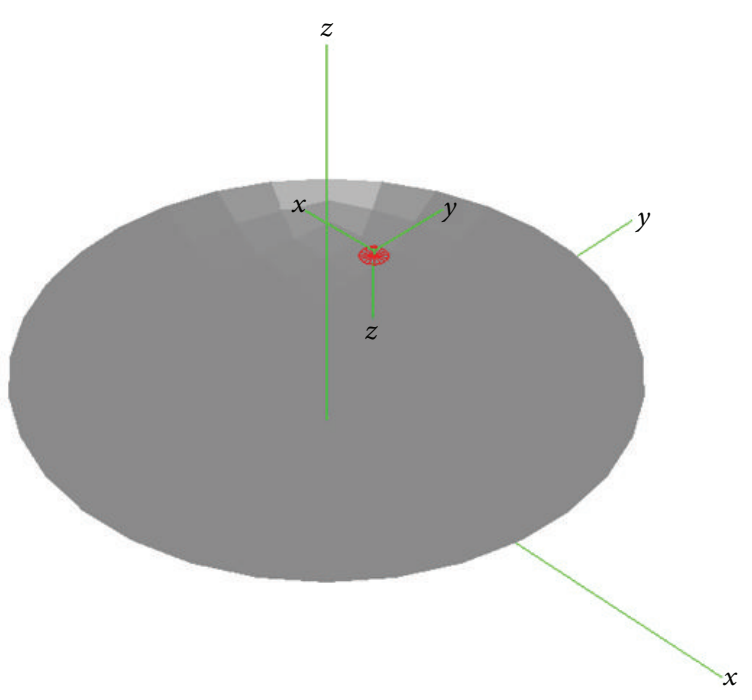

Figure 21: Single reflector with the horn shifted.

(ii) In the second one, it is considered a 2D array consisting of nine horns.

The horns used in these situations are the same as in previous study, whose radiation pattern was shown in Figure 17 , and the frequency of the simulation is also the same, $10 \mathrm{GHz}$.

5.2.1. Linear Array over $x$-Axis. The reflector of Figure 23 is illuminated by an array of three horns that are separated $2 \lambda$; so, their positions are

$$
(-0.02,0.0,0.4), \quad(0.0,0.0,0.4), \quad(0.02,0.0,0.4) \text {. }
$$

The radiation pattern of this reflector has been obtained applying GTD and MoM. Figure 24 shows the results for the cut in $\Phi=0^{\circ}$. The second and main lobes are located at $\theta=2^{\circ}$, $\theta=-2^{\circ}$, and $\theta=0^{\circ}$, respectively, due to the three horns.

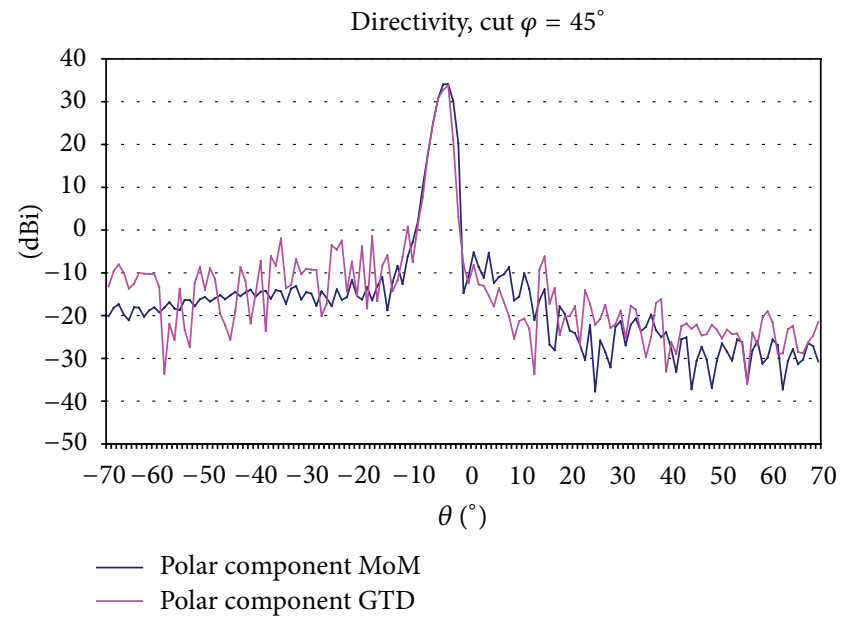

FIGURE 22: Polar component obtained with MoM and GTD, cut in phi $=45$.

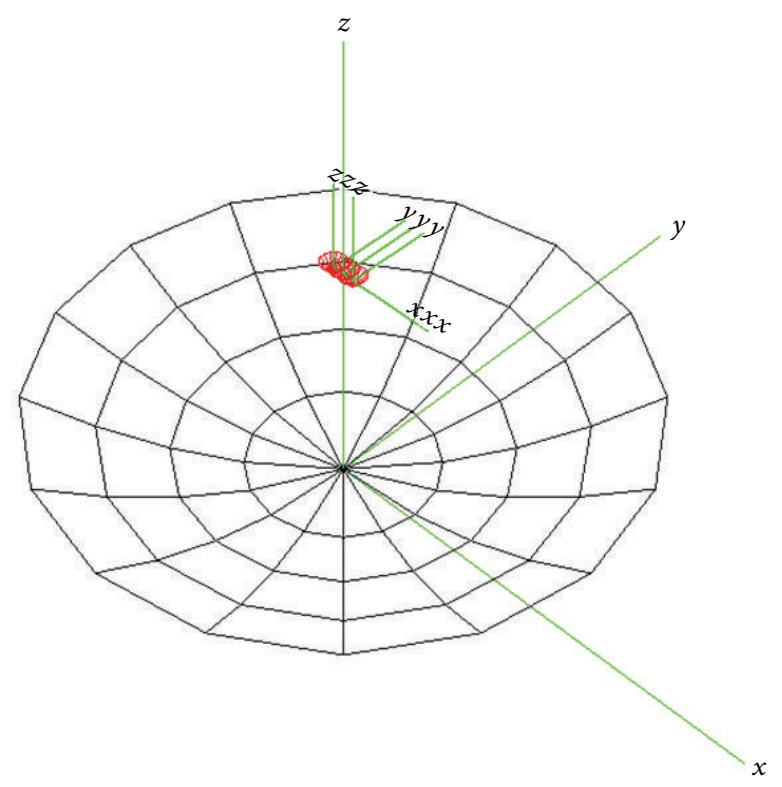

FIGURE 23: Single reflector feed with a linear array of horns located over the $x$-axis.

5.2.2. 2D Array. The reflector presented in Figure 25 has been fed through an array of two dimensions in which the antennas are separated $2 \lambda$; so, their positions are

$$
\begin{array}{ccc}
(-0.02,0.02,0.4), & (0.0,0.02,0.4), & (0.02,0.02,0.4) \\
(-0.02,0.0,0.4), & (0.0,0.0,0.4), & (0.02,0.0,0.4) \\
(-0.02,-0.02,0.4), & (0,-0.02,0.4), & (0.02,-0.02,0.4)
\end{array}
$$

The radiation pattern of the antennas array is the one shown in Figure 17. The cut in $\varphi=0^{\circ}$ is represented in Figure 26. The side and the main lobes are identified at $\theta=2^{\circ}$, $\theta=-2^{\circ}$, and $\theta=0^{\circ}$, respectively. The three horns located on the $x$-axis account for these lobes. 


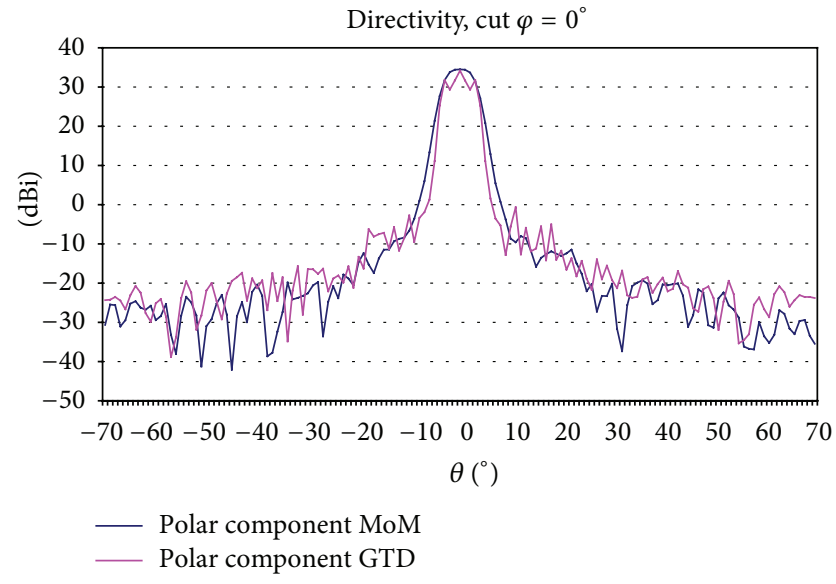

Figure 24: Polar component obtained with MoM and GTD, cut in phi $=0$.

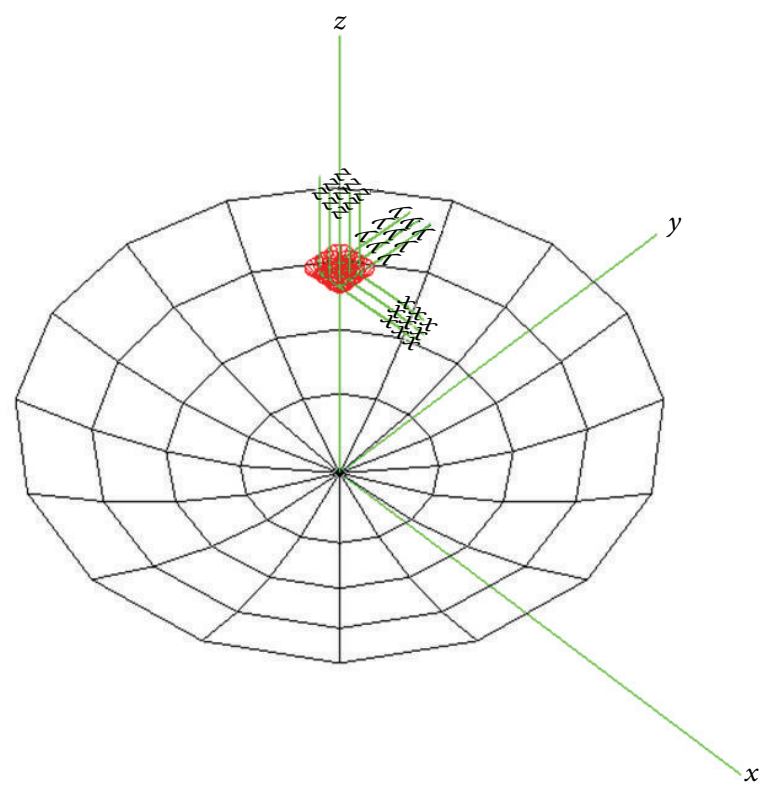

FIGURE 25: Single reflector feed with a 2D array of horns.

Figure 27 depicts the results for the cut in $\varphi=90^{\circ}$. The side and main lobes are seen at $\theta=2^{\circ}, \theta=-2^{\circ}$, and $\theta=0^{\circ}$, respectively, due to the horns located on the $y$-axis.

The simple cases shown in this section cannot be analysed applying GTD without Master Points technique and the new distance function shown in Section 4. All of them have been run in a PC with an Intel Core 2 Duo (only one core was used) at $1.87 \mathrm{GHz}$.

5.3. Blocking Produced by an Obstacle. Another interesting effect to study is the hiding part of the radiation pattern caused by an obstacle placed over the aperture of the antenna. Two different scenarios have been considered. The first one is a simple case composed of a single reflector with an obstacle located over its aperture. The second one is more complicated;

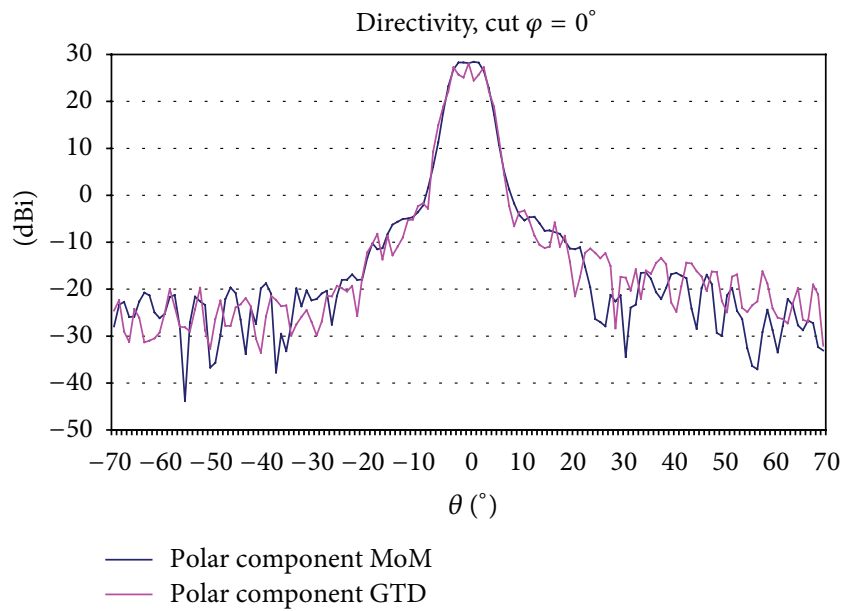

FIgURE 26: Polar component obtained with MoM and GTD, cut in phi $=0$.

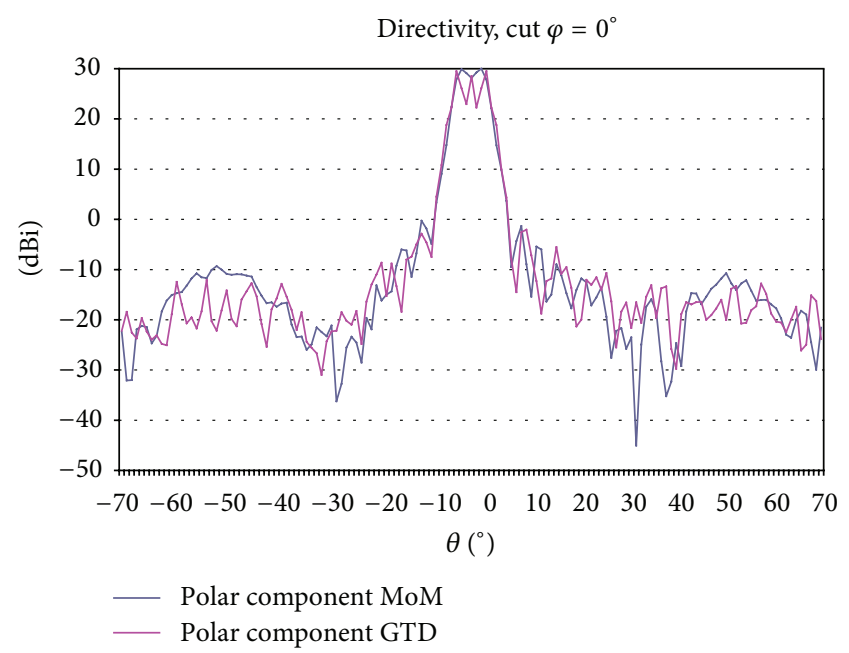

FIgUre 27: Polar component obtained with MoM and GTD, cut in phi $=90^{\circ}$.

the geometrical model of a reinforced has been analyzed shifting the reflector on its roof.

5.3.1. Calculating the Directivity of a Reflector with an Obstacle. The scenario shown in Figure 28 is considered. The reflector is fed by a single rectangular horn, whose radiation pattern remains the same as in Figure 17, and placed in the focus of the reflector. As it is presented in Figure 28, the obstacle is hiding approximately $34 \%$ of the aperture of the reflector.

Figure 29 shows the effects produced by the obstacle. So, the graph obtained for the single reflector without the obstacle, scenario shown in Figure 16, has been compared with the graph of the reflector with the obstacle, scenario shown in Figure 28. The results of both scenarios have been obtained with the high frequency technique, GTD-Master Points, and the rigorous method, MoM.

The main consequence generated by the obstacle is the growth of the level of the secondary lobes. 


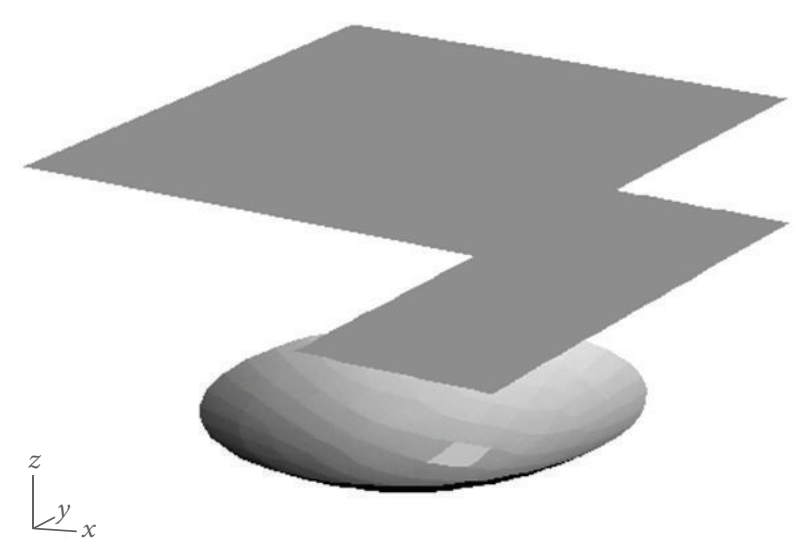

FIGURE 28: Single reflector with an obstacle and the observation surface.

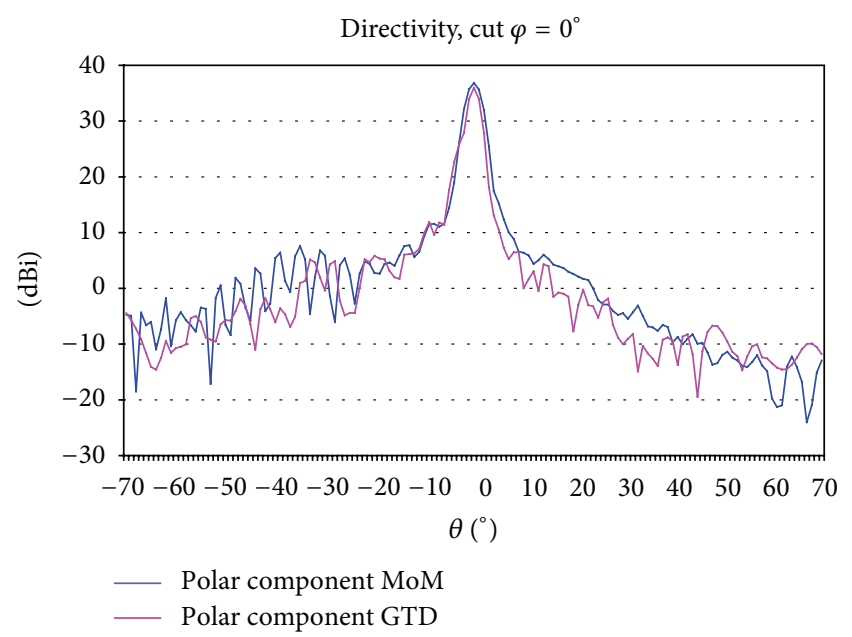

Figure 29: Polar component obtained with MoM and GTD, cut in phi $=0$.

5.3.2. Calculating the Directivity of a Reflector Located on a Vehicle. Figure 30 shows the geometric model of an armored vehicle built with both flat and curved surfaces. A reflector is on the roof of the vehicle, whose radiation axis is the $z$-axis.

The study of this scenario has been done at $12 \mathrm{GHz}$, and the reflector is fed with the horn shown in Figure 17, located on its focus $(6.758,2.1,3.04)$. The observation surface is placed over the aperture of the reflector. It has been sampled at a frequency of $\lambda / 3$, which means getting the near field in a total of 3,660 observation points. The results for the model of Figure 30 have been obtained applying GTD and MoM. Figure 31 shows the graph for a cut in $\varphi=0^{\circ}$ and sweep from $\theta=-70^{\circ}$ to $\theta=70^{\circ}$.

This simulation has been done in an Intel Xeon at $2.13 \mathrm{GHz}$. The Table 1 compared the CPU time consumed in the analysis when GTD-Master Points and MoM techniques are applied.

If a turn of $90^{\circ}$ in $\varphi$ and $145^{\circ}$ in $\theta$ is applied to the reflector of the armored vehicle, the new geometrical model is shown in Figures 32 and 33. This represents an interesting

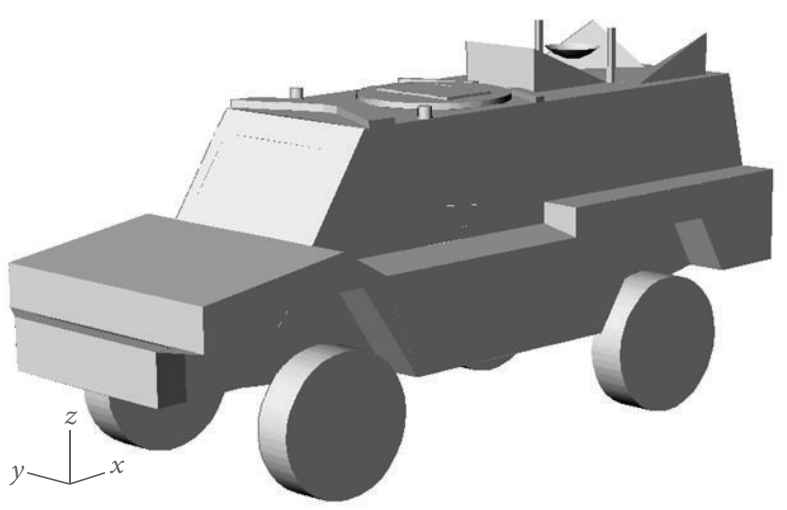

FIGURE 30: Geometrical model of an armored vehicle.

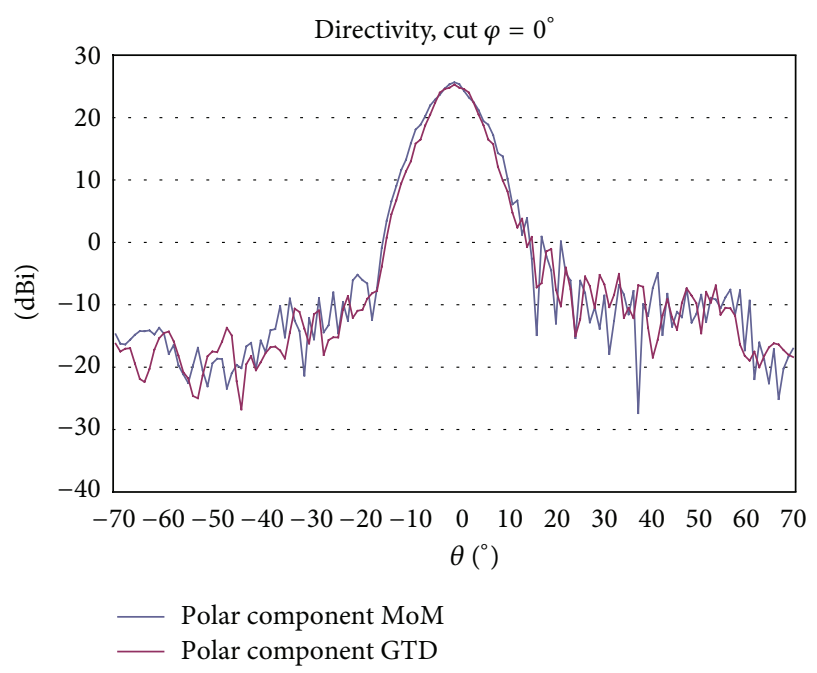

FIgUre 31: Polar component obtained with MoM and GTD, cut in $\varphi=0^{\circ}$.

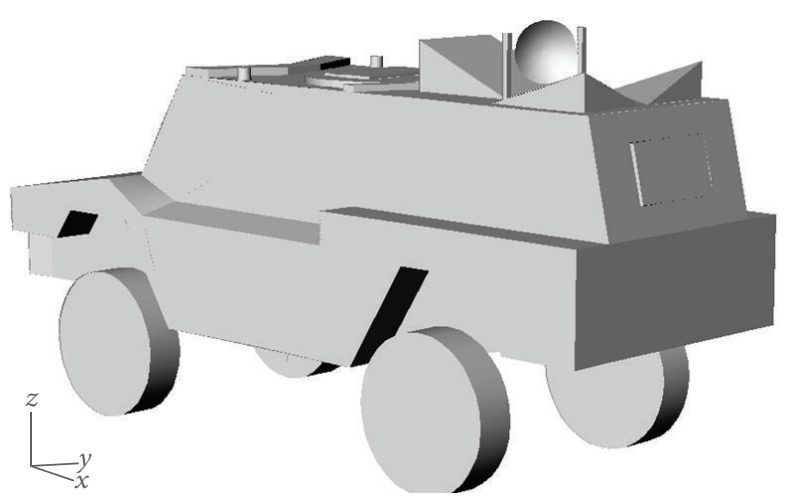

FIGURE 32: Geometrical model of the reinforced car with its reflector shifted.

case because of the blockade that will cause one of the parts of the roof. Now, the $z$-axis is not the radiation axis of the reflector.

The simulation has been done feeding the reflector with a rectangular horn placed at the focus of the reflector $(6.93,2.083,3.2)$ and at the same frequency as in the previous 


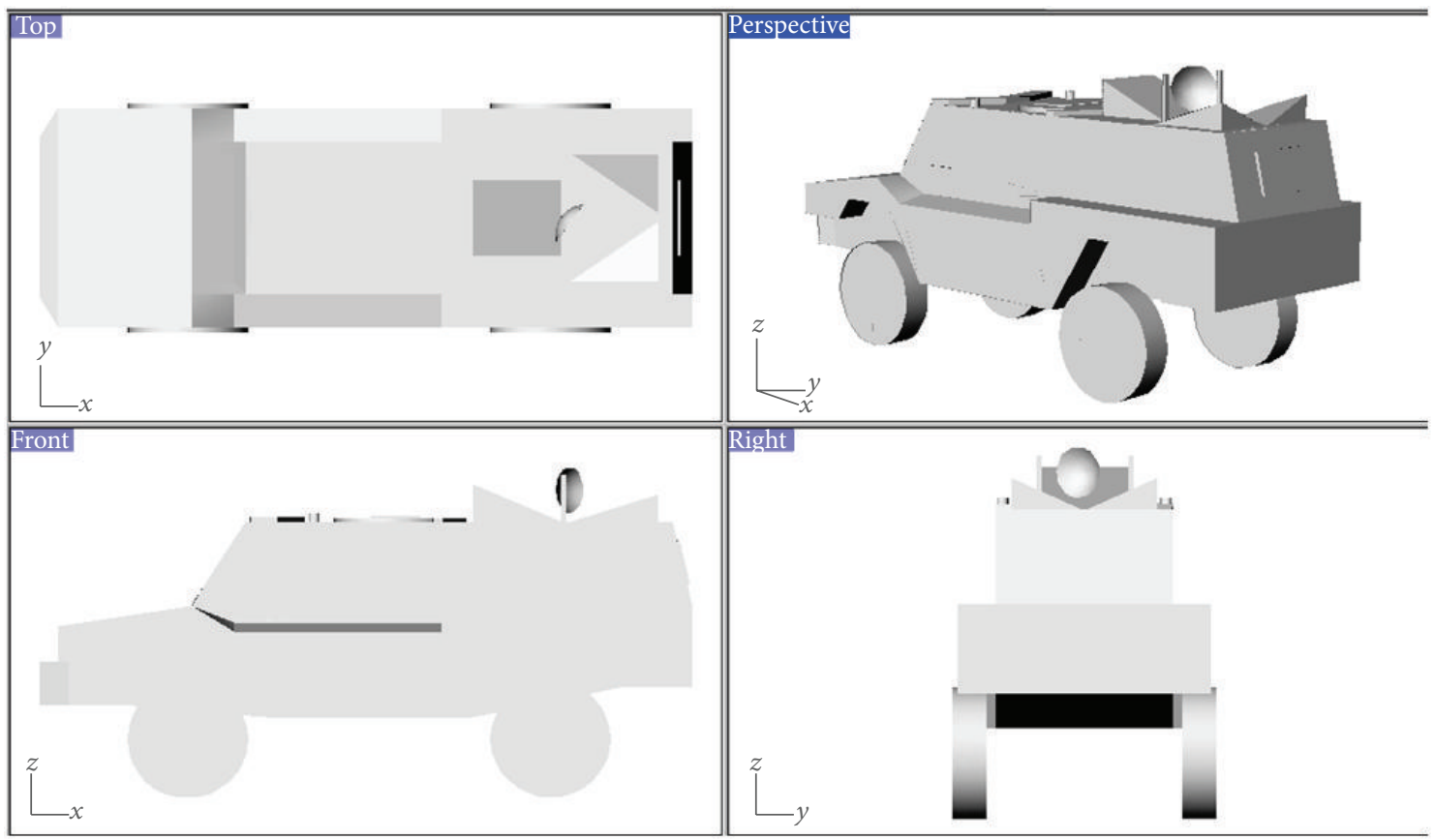

FIGURE 33: Different views of the reinforced vehicle.

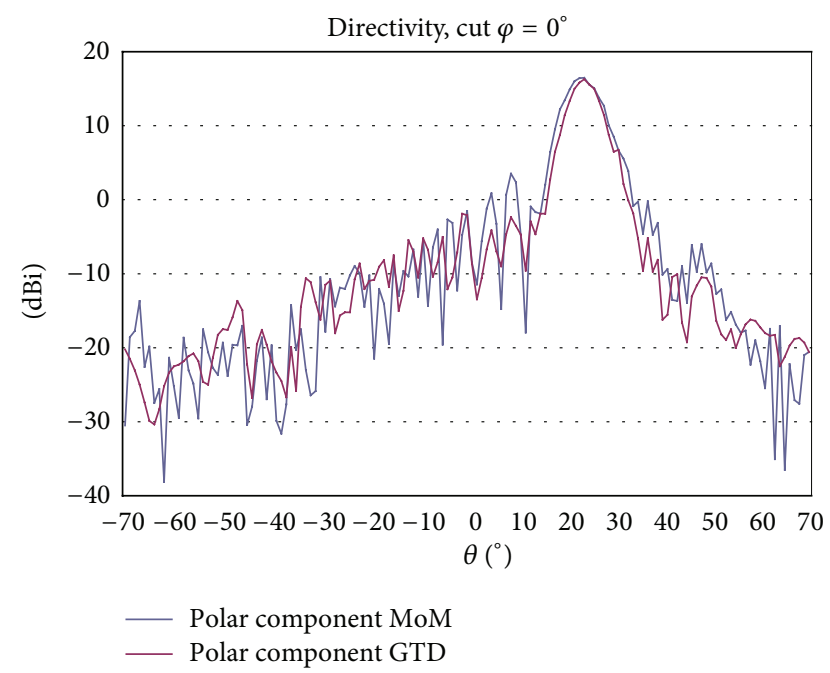

FIGURE 34: Polar component obtained with GTD and MoM, cut in $\varphi=0^{\circ}$.

TABLE 1: Comparison of the CPU time need for the analysis when GTD-Master Points and MoM techniques are applied.

\begin{tabular}{lcc}
\hline & GTD-Master Points & MoM \\
\hline CPU time & $47 \mathrm{~min}$. & $7 \mathrm{~h} .14 \mathrm{~min}$. \\
\hline
\end{tabular}

case, $12 \mathrm{GHz}$. The sampling frequency of the observation surface is $\lambda / 3$. The results obtained with the shifted reflector have been calculated applying MoM and GTD (Figure 34). Because of the cancellation of the directivity caused by some part of the roof, the level of the second lobes has
TABLE 2: Comparison of the CPU time need for the analysis when GTD-Master Points and MoM techniques are applied.

\begin{tabular}{lcc}
\hline & GTD-Master Points & MoM \\
\hline CPU time & $53 \mathrm{~min}$. & $9 \mathrm{~h} .41 \mathrm{~min}$. \\
\hline
\end{tabular}

been increased. This is the main consequence of locating an obstacle in the directivity of the antenna.

This simulation has been done in an Intel Xeon at $2.13 \mathrm{GHz}$. As shown in Table 2, the CPU time consumed by MoM is higher than the CPU time consumed by GTD-Master Points.

\section{Conclusion}

This paper presents the improvements developed to analyze of the radiation pattern arbitrarily shaped and fed reflector antennas. Different techniques can be applied to perform this analysis. In particular, the Geometrical Theory of Diffraction is considered in this paper. Although this technique is very useful to compute the far field radiated by these structures, it has the drawback of being very time consuming. Therefore, the new technique Master Points has been developed to speed up this process, since it reduces the number of times in which the ray tracing is calculated.

A complete study of the radiation pattern of a parabolic reflector fed with a horn or an array of horns and a study of the far field pattern degradation caused by an obstacle have been presented. The results obtained with the new method have been compared with MoM showing good agreement. Thus, it can be concluded that the new function for the CGM and the combination of GTD with Master Points represent a 
good alternative to obtain an accurate and fast evaluation of the radiation pattern of reflector antennas.

\section{Acknowledgments}

This work has been supported in part by the Comunidad de Madrid Project S-2009/TIC1485, the Castilla-La Mancha Project PPII10-0192-0083, and the Spanish Department of Science, Technology Projects TEC2010-15706 and CONSOLIDER-INGENIO no. CSD-2008-0068.

\section{References}

[1] J. S. Brown, "Unidirectional antennas for 450 to $460 \mathrm{mc}$, Transactions of the IRE Professional Group on Vehicular Communications, vol. 1, no. 1, pp. 134-140, 1952.

[2] C. C. Cutler, "Parabolic-antenna design for microwaves," Proceedings of the IRE, vol. 35, no. 1, pp. 1284-1294, 1947.

[3] S. Seely, "Microwave antenna analysis," Proceedings of the IRE, vol. 35, no. 10, pp. 1092-1095, 1947.

[4] O. M. Conde, J. Pérez, and M. F. Cátedra, "Stationary phase method application for the analysis of radiation of complex 3D conducting structures," IEEE Transactions on Antennas \& Propagation, vol. 49, no. 5, pp. 724-731, 2001.

[5] R. G. Kouyoumjiam, "Asymptotic high-frequency methods," Proceedings of the IEEE, vol. 53, pp. 864-876, 1965.

[6] F. Vico-Bondia, M. Ferrando-Bataller, and A. Valero-Nogueira, "A new fast physical optics for smooth surfaces by means of a numerical theory of diffraction," IEEE Transactions on Antennas and Propagation, vol. 58, no. 3, pp. 773-789, 2010.

[7] M. S. Narashimhan and K. M. Prasad, "GTD analysis of the near-field patterns of a prime-focus symmetric paraboloidal reflector antenna," IEEE Transactions on Antennas and Propagation, vol. 29, no. 6, pp. 959-961, 1981.

[8] Y. Rahmat-Samii and V. Galindo-Israel, "Shaped reflector antenna analysis using the Jacobi-Bessel series," IEEE Transactions on Antennas and Propagation, vol. 28, no. 4, pp. 425-435, 1980.

[9] W. L. Ko, R. Mittra, and S. W. Lee, "Aperture blockage in reflector antennas," IEEE Transactions on Antennas and Propagation, vol. 32, no. 3, pp. 282-287, 1984.

[10] A. Boag and C. Letrou, "Fast radiation pattern evaluation for lens and reflector antennas," IEEE Transactions on Antennas and Propagation, vol. 51, no. 5, pp. 1063-1068, 2003.

[11] S. W. Lee, P. Cramer, K. Woo, and Y. Rahmat-Samii, "Diffraction by an arbitrary subreflector: GTD solution," IEEE Transactions on Antennas and Propagation, vol. 27, no. 3, pp. 305-316, 1979.

[12] W. H. Press, B. P. Flannery, S. A. Teukolsky, and W. T. Vetterling, Numerical Recipes, Cambridge University Press, Cambridge, UK, 1987.

[13] S. Y. Tan and H. S. Tan, "A microcellular communications propagation model based on the uniform theory of diffraction and multiple image theory," IEEE Transactions on Antennas and Propagation, vol. 44, no. 10, pp. 1317-1326, 1996.

[14] G. Farin, Curves and Surfaces for Computer Aided Geometric Design, Academic Press, 1988.

[15] W. Dahmen, M. Gasca, and C. A. Micchelli, Eds., Computation of Curves and Surfaces, Kluwer Academic Publishers, 1990.

[16] D. A. McNamara, C. W. I. Pistorius, and J. A. G. Malherbe, Introduction to the Uniform Geometrical Theory of Diffraction, The Artech House Microwave, Norwood, Mass, USA, 1989. 

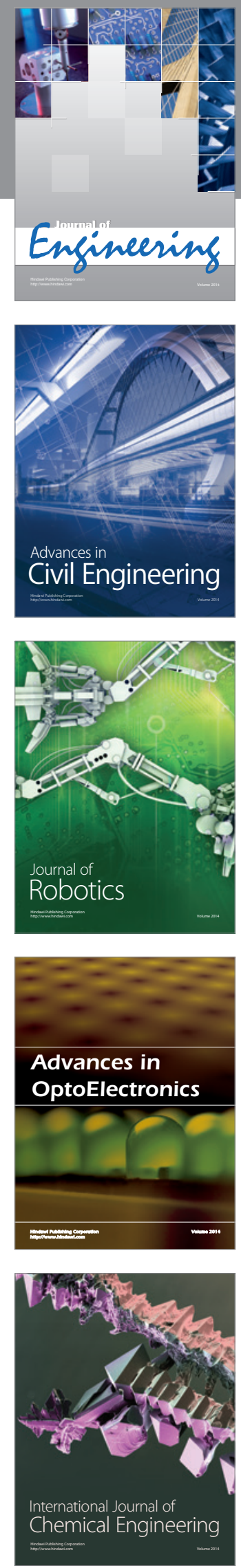

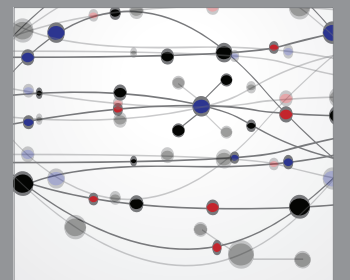

The Scientific World Journal
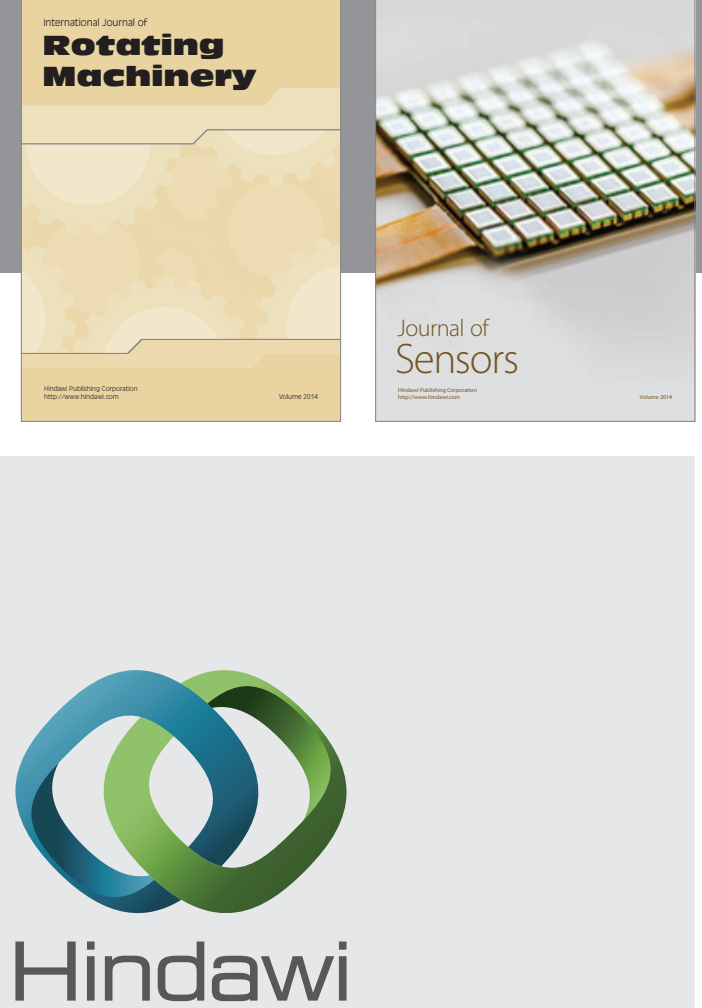

Submit your manuscripts at http://www.hindawi.com
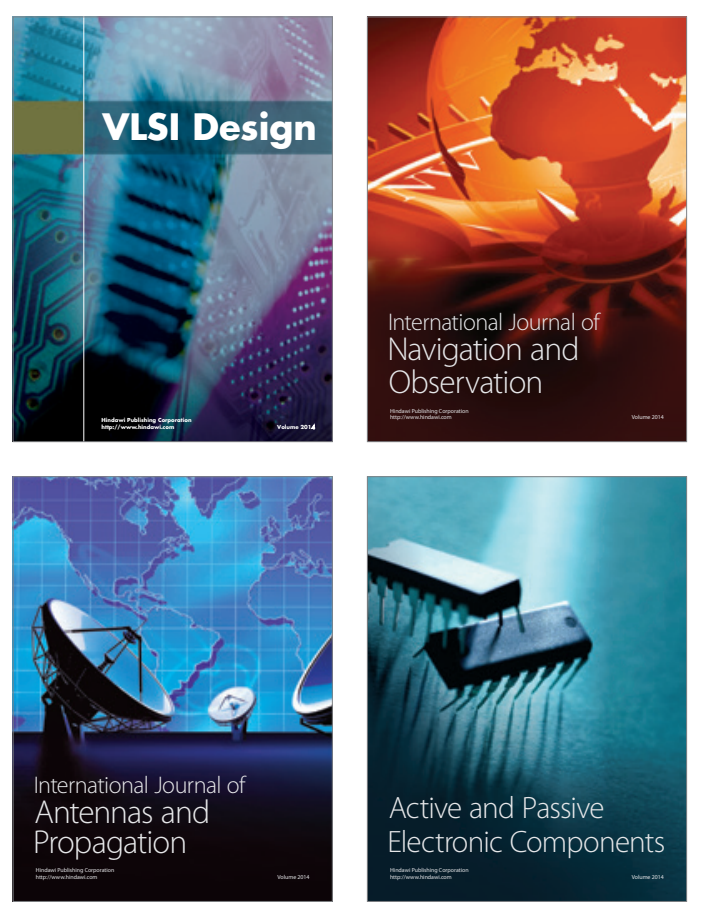
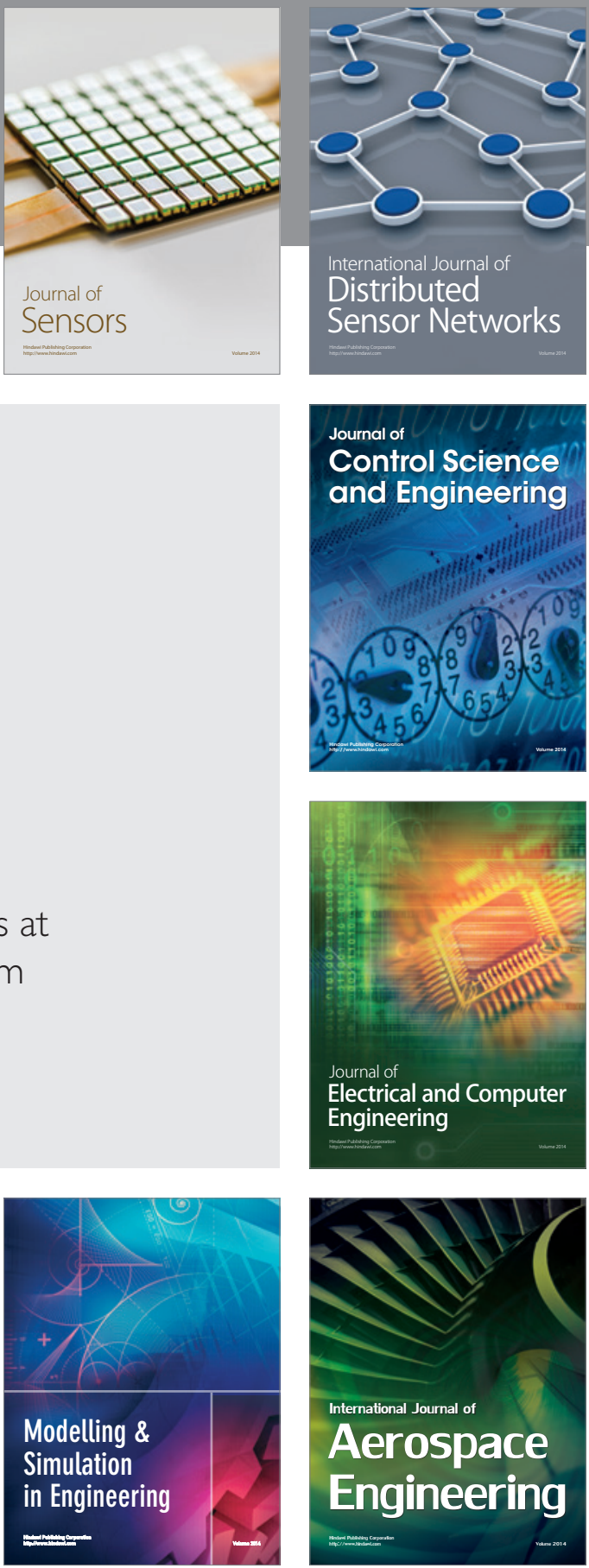

Journal of

Control Science

and Engineering
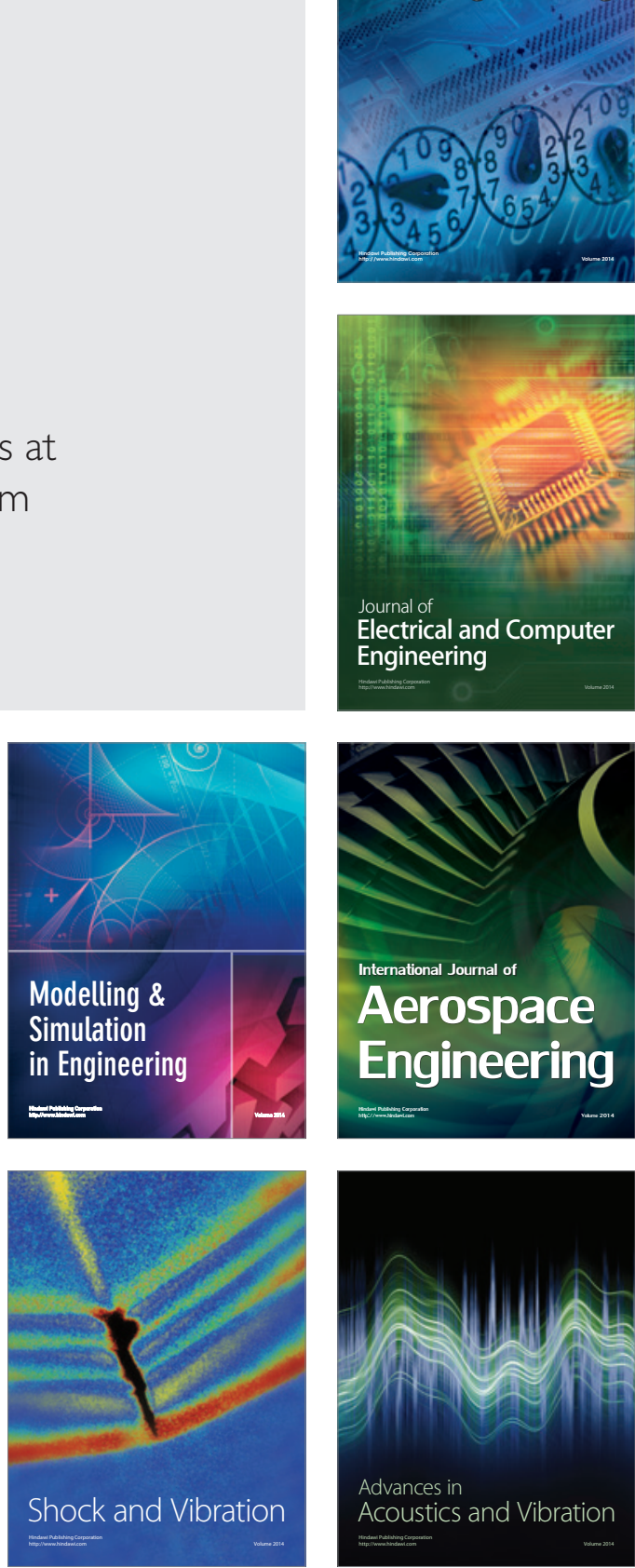